\title{
Entrelacs
}

Cinéma et audiovisuel

\section{Figures de la (pro)création : arachnides et autres symboles chez Alejandro Jodorowsky}

Jules Fontaine et Marion Sergent

\section{(2) OpenEdition}

\section{Journals}

Édition électronique

URL : http://journals.openedition.org/entrelacs/5591

DOI : 10.4000/entrelacs.5591

ISSN : 2261-5482

Éditeur

Éditions Téraèdre

Référence électronique

Jules Fontaine et Marion Sergent, «Figures de la (pro)création : arachnides et autres symboles chez Alejandro Jodorowsky », Entrelacs [En ligne], 16 | 2019, mis en ligne le 06 novembre 2019, consulté le 03 décembre 2019. URL : http://journals.openedition.org/entrelacs/5591; DOI : 10.4000/entrelacs. 5591

Ce document a été généré automatiquement le 3 décembre 2019

Tous droits réservés 


\title{
Figures de la (pro)création : arachnides et autres symboles chez Alejandro Jodorowsky
}

\author{
Jules Fontaine et Marion Sergent
}

\section{Introduction}

1 Qu'il soit cinématographique, littéraire ou plastique, le langage d'Alejandro Jodorowsky s'appuie sur la richesse d'interprétation des signes symboliques, ou comme il l'écrivait : «Un symbole ne transmet pas un message précis, il agit comme un miroir qui reflète le niveau de conscience du chercheur ${ }^{1}$.» Dans cette perspective, sa création artistique n'est pas sans lien avec ses pratiques thérapeutique et divinatoire. La psychomagie s'appuie en effet sur une mise en scène d'actes symboliques afin d'aider le consultant à résoudre un conflit inconscient, tandis que le tarot repose sur l'interprétation des lames au symbolisme complexe. Si Ferdinand de Saussure remarquait dans ses Cours de linguistique générale qu'un symbole «a pour caractère de n'être jamais complètement arbitraire » et qu'« il y a un rudiment de lien entre idée et signe ${ }^{2} »$, il ne spécifie pas la nature de cette relation. La constitution analogique du symbole peut aussi bien appeler le vécu individuel et biographique que le contexte culturel et social, comme le démontre Émilie Granjon ${ }^{3}$. En ce sens, le contenu que cache la forme symbolique de l'araignée est lié aussi bien à l'histoire personnelle de Jodorowsky qu'à un inconscient collectif. Au fil de son œuvre protéiforme, les images arachnéennes surgissent régulièrement dans un réseau de problématiques en relation avec la question de la création. Comment parviennent-elles à soulever la richesse sémantique d'une telle notion? La réponse semble se trouver dans la diversité des figures qu'elles convoquent. 


\section{L'œuvre au bout du fil}

2 Cette conception symbolique de l'œuvre d'art se retrouve précisément dans un recueil de petites histoires intitulé Les Araignées sans mémoire et autres fables paniques. Le premier texte qui donne son nom à l'ouvrage narre l'amnésie qui toucha un jour les araignées quant à leur capacité à créer des toiles nécessaires à leur survie. Elles deviennent sans raison incapables d'agencer la soie qui sort de leurs filières. S'adaptant à leur nouvelle condition, elles se constituent alors en société souterraine et continuent de tisser, sans comprendre le sens de leur geste. Quelques-unes parviennent à tirer profit de cette faculté.

Certaines se proclamèrent alors « artistes » et commencèrent à tisser des objets qui rappelaient vaguement les anciennes toiles. Elles n'étaient ni rondes, ni fines, ni visqueuses, incapables d'attraper le plus petit insecte, mais remplissaient d'orgueil leurs créatrices ventrues. La mélancolie de l'amnésie s'installant, les araignées placèrent bientôt ces approximations de toiles, ces arachnéennes tentatives, sur des autels familiaux et se mirent à les vénérer. Avec le cours du temps, elles les entassèrent dans des musées puis dans des temples. On finit par les appeler des « symboles $»^{4}$.

3 La toile devient la trace d'un savoir instinctif passé mais oublié, acquérant alors un caractère divin. Les araignées sentent bien que derrière ces créations il y a une signification qu'elles ne parviennent pas à retrouver, tapie dans l'inconscient collectif. Si la fable se termine sur une araignée ayant recouvré la mémoire, tissant sur la place publique une toile, cet esprit lucide finit sous les injures de ses consœurs et sa création est détruite. Cette métaphore anthropomorphique offre une critique de l'enfermement de l'œuvre d'art dans un espace intérieur consacré, alors que sa place véritable est en extérieur, à la vue de toutes. Elle met aussi en avant le caractère orgueilleux de l'artiste, fier de sa création en dépit de son imperfection et s'arrogeant une supériorité alors que toutes les araignées sont capables de tisser. Comme Sylvie Ballestra-Puech le remarquait, le propos de Jodorowsky est nettement politique, esquissant l'édification d'un "pouvoir abusif et arbitraire ${ }^{5}$ ». Derrière cette fable c'est bien la question du statut de l'artiste qui est en jeu: est-ce la personne qui s'auto-proclame ainsi ou bien celle qui parvient à retrouver un sens caché ?

La figure arachnéenne est réutilisée dans une autre fable de ce même recueil, nommée "L'Araignée ambitieuse " ${ }^{6}$. Cette fois la protagoniste s'élance à la recherche d'une perfection inatteignable, voulant enfermer des aigles dans ses filets. C'est bien grâce à sa toile, c'est-à-dire l'œuvre artistique, qu'elle souhaite attraper un être hors de sa portée. L'ambition démesurée de cette araignée-artiste ne peut qu'aboutir à une fin funeste : l'animal est dévoré par l'objet de sa convoitise. Cette histoire de l'araignée ambitieuse a également été traitée dans une planche dessinée, autre format des fables paniques de Jodorowsky, imprimée le 8 décembre 1968 dans la revue El Heraldo de México, soit bien avant la parution de l'ouvrage de 1980. On peut y lire une formule assez éloquente sur les ambitions de l'araignée : «Elle se désintéressa de la qualité de ses toiles et fit de la chasse à l'aigle l'unique objectif de sa vie ${ }^{7}$ ». Mue par un idéal, celle-ci délaisse son art du tissage, source de son alimentation, pour ne dédier ses forces qu'à la conquête de l'animal céleste, avant de devenir elle-même la nourriture de l'aigle. Si l'araignée s'est sentie humiliée lorsque l'oiseau déchira sa toile, la fable n'indique pas si c'est dans une extrême lucidité ou un accès de folie qu'elle décide de renoncer à sa vie terrestre pour s'unir avec cette figure divine. Jodorowsky n'offre pas 
une lecture moraliste, les derniers mots de l'araignée laissant une certaine ambiguïté : "J'ai chassé un aigle! Je pénètre son sang et sa chair! Je suis en train de devenir un aigle ${ }^{8}$ ». Certes, il y a un acte de transsubstantiation, mais en continuant d'affirmer son individualité en opposition au rapace, n'est-ce pas l'annihilation de son être qui l'attend? L'analyse de Sylvie Ballestra-Puech confirme cette interprétation ouverte, oscillant entre l'image d'une démesure aliénante et d'une fusion libératrice ${ }^{9}$.

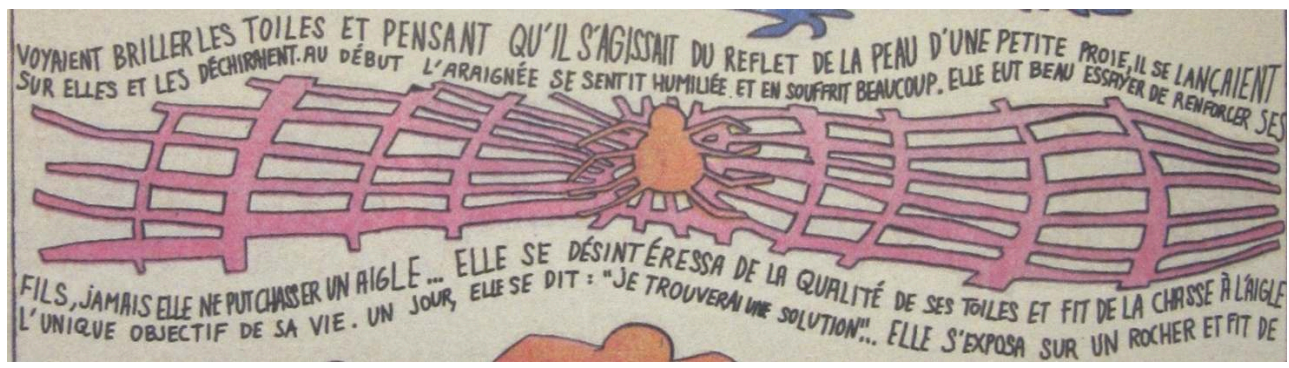

Alejandro Jodorowsky, «Fable panique », détail, El Heraldo de México, 8 décembre1968 reproduit dans Fables Paniques, Arles : Actes Sud, 2017

5 Là encore, Jodorowsky offre à travers cette image de l'araignée une réflexion sur la figure de l'artiste en des termes très proches des débats néo-platoniciens de la Renaissance qui, par analogie entre macrocosme et microcosme comparaient la puissance du peintre à l'acte de création divine ${ }^{10}$. L'araignée est une véritable créatrice, produisant sa toile ex-nihilo, ce qui la rapproche bien de ce Dieu avec lequel elle veut se fondre. En affirmant qu'elle devient oiseau, elle nous donne la métaphore de l'artiste qui ne se distingue plus du Créateur. Comme Jodorowsky l'énonce dans un entretien de 1989, "Dieu est le grand artiste ${ }^{11}$ ». D'ailleurs c'est une telle équivalence entre la créativité humaine et divine qui est au cœur du mythe ovidien d'Arachné, auquel renvoie cette histoire de l'araignée ambitieuse. Tisseuse de grande renommée trop orgueilleuse de son talent, Arachné s'attire la colère de la déesse Pallas ${ }^{12}$. Alors qu'elles s'affrontent dans l'art du tissage, c'est-à-dire dans leur puissance créatrice, la divinité frappe Arachné après avoir découvert sa tapisserie dépeignant les crimes des dieux. Humiliée par ce geste, la jeune femme se suicide, pendue à l'un de ses fils. Pallas lui refuse la mort et préfère la transformer en araignée.

6 Ce thème de l'arrogance née du pouvoir créateur se retrouve dans une autre planche publiée le 5 juillet 1970 et mettant en scène Jodorowsky lui-même ${ }^{13}$. L'artiste se représente en marionnettiste capricieux, sectionnant les fils de son pantin à la manière d'Atropos, la Moire qui coupe le fil de vie, avant de le balayer tel un vulgaire détritus. Cet acte assez violent s'appuie sur l'opposition entre un sujet et un objet liés par une relation de possession, comme l'indique la première phrase « $\mathrm{j}$ 'avais une marionnette ${ }^{14}$ ». Exerçant sa volonté, même destructrice, sur une version réduite et inanimée de luimême - la marionnette porte des vêtements identiques -, le personnage de Jodorowsky finit par subir un sort analogue. Les fils invisibles qui maintenaient son corps l'abandonnent et un balai géant apparaît dans le ciel pour le chasser. Alors qu'il a la faculté de donner vie à son fantoche en actionnant les fils, l'artiste exerce son pouvoir démiurgique de manière inconséquente, en rejetant la marionnette à son statut d'objet. Croyant en sa supériorité, l'artiste voit ensuite son libre arbitre mis à mal par des forces célestes. Cet autoportrait en marionnettiste trouve de surcroît un écho dans la biographie de l'artiste. Alors qu'il est âgé d'une vingtaine d'années, Jodorowsky fabrique de nombreuses marionnettes puis monte des spectacles au sein de l'université du Chili, avec sa propre compagnie El Bululú ${ }^{15}$. 


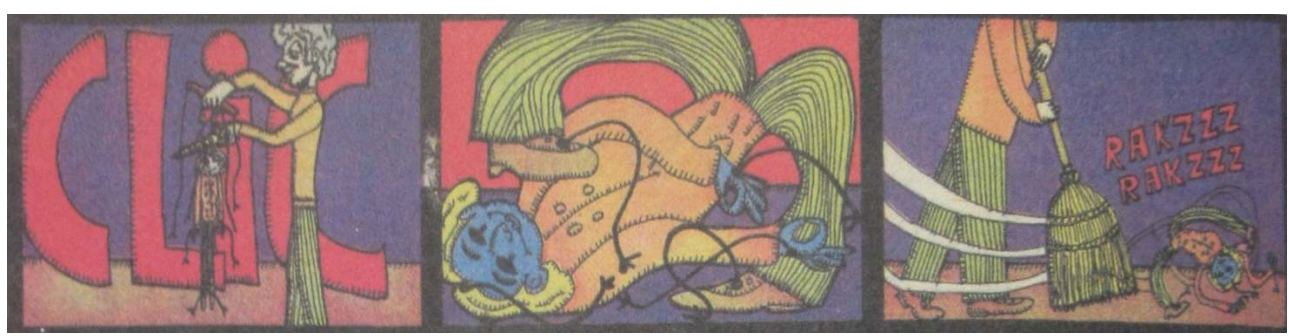

Alejandro Jodorowsky, "Fable panique », détail, El Heraldo de México, 5 juillet 1970 reproduit dans Fables Paniques, Arles : Actes Sud, 2017

7 Il y a une mise en abyme dans cette fable: Jodorowsky en artiste-marionnettiste manifeste sa domination sur un avatar de moindre dimension, avant d'être balayé par une force plus grande qui est celle de Jodorowsky, l'auteur de cette histoire dessinée. L'usage d'un discours auto-réflexif est fréquent dans son œuvre, Jodorowsky se mettant lui-même en scène dans une posture de toute-puissance à plusieurs reprises. Cette représentation incarne une image du pouvoir de l'artiste qui, dans le cas du marionnettiste, est employé à mauvais escient. La même image se retrouve dans le premier long-métrage de Jodorowsky réalisé en 1968. Fando et Lis (Fando y Lis) est une adaptation très libre d'une pièce de Fernando Arrabal qui donne à voir la quête difficile d'un couple pour rejoindre Tar, un monde où tout est possible. Jodorowsky apparaît en marionnettiste coupant un à un les liens de son pantin, pour ensuite le balayer. L'interprétation d'un démiurge impitoyable est confirmée dans les commentaires qu'il fait de cette scène :

L'incarnation de Dieu apparaît, une marionnette à la main. Il va couper les fils de la marionnette, il la dirige. J'ai toujours voulu jouer Dieu. Dans El Topo je dis : « Je suis Dieu ». Lui, c'est Dieu. C'est une scène de mise à mort. Dieu est le plus grand tueur en série. Le plus grand. Qu'est-ce qu'un être humain ? Rien ${ }^{16}$.

Cependant, la marionnette n'est pas seulement une créature pleinement soumise aux désirs de celui qui l'anime, mais peut acquérir une vie propre. En effet, ce renversement est au cœur de L'École des ventriloques, une pièce théâtrale écrite en 2002, puisque ce sont les marionnettes qui contrôlent les humains ${ }^{17}$. Jodorowsky a par ailleurs écrit à ce sujet dans Le Théâtre de la Guérison:

J'ai d'emblée vu en la marionnette une figure hautement métaphysique. Tout d'abord, j'étais fasciné de voir un objet fabriqué de mes propres mains m'échapper. Dès que je mettais ma main dans la marionnette pour l'animer, le personnage se mettait à vivre de manière quasi autonome. [...] Au lieu d'être un créateur, il me semblait faire office de serviteur ${ }^{18}$.

Cette citation est, à elle seule, un parfait résumé de l'intrigue du sixième long métrage de Jodorowsky, Santa Sangre réalisé en 1989. L'histoire est celle de Fenix (Axel Jodorowsky) poussé au féminicide par sa marionnette qui n'est autre que la transposition de sa mère décédée durant son enfance. Pourtant, à la manière du personnage illusionné, le spectateur n'est pas censé savoir qu'il s'agit d'un pantin avant le dénouement du film, car ce qui apparaît véritablement à l'écran c'est la mère, Concha (Blanca Guerra), femme amputée des deux bras. Placé constamment dans l'ombre de sa marionnette, Fenix se résume à ses bras et rien d'autre. Le marionnettiste a abandonné les fils pour s'engouffrer de tout son corps à l'intérieur de sa marionnette, sacrifiant au passage sa propre existence. Il fait don de ses membres à sa mère afin qu'elle puisse tricoter ou jouer du piano dans un asservissement total. Cet effacement absolu du créateur au profit de sa création le vampirise au point d'annihiler sa 
personnalité et jusqu'à sa propre existence. C'est pour cette raison que Fenix s'identifie à l'homme invisible, héros du film éponyme de James Whale ${ }^{19}$ dont on voit un extrait, ce que Concha s'empresse de dénigrer violemment comme paroxysme de l'intrusion d'une mère dans la sphère intime de son fils : "Sans moi tu n'es rien. Personne ne te voit et personne ne te remarque. Tout comme ton héros ridicule ».

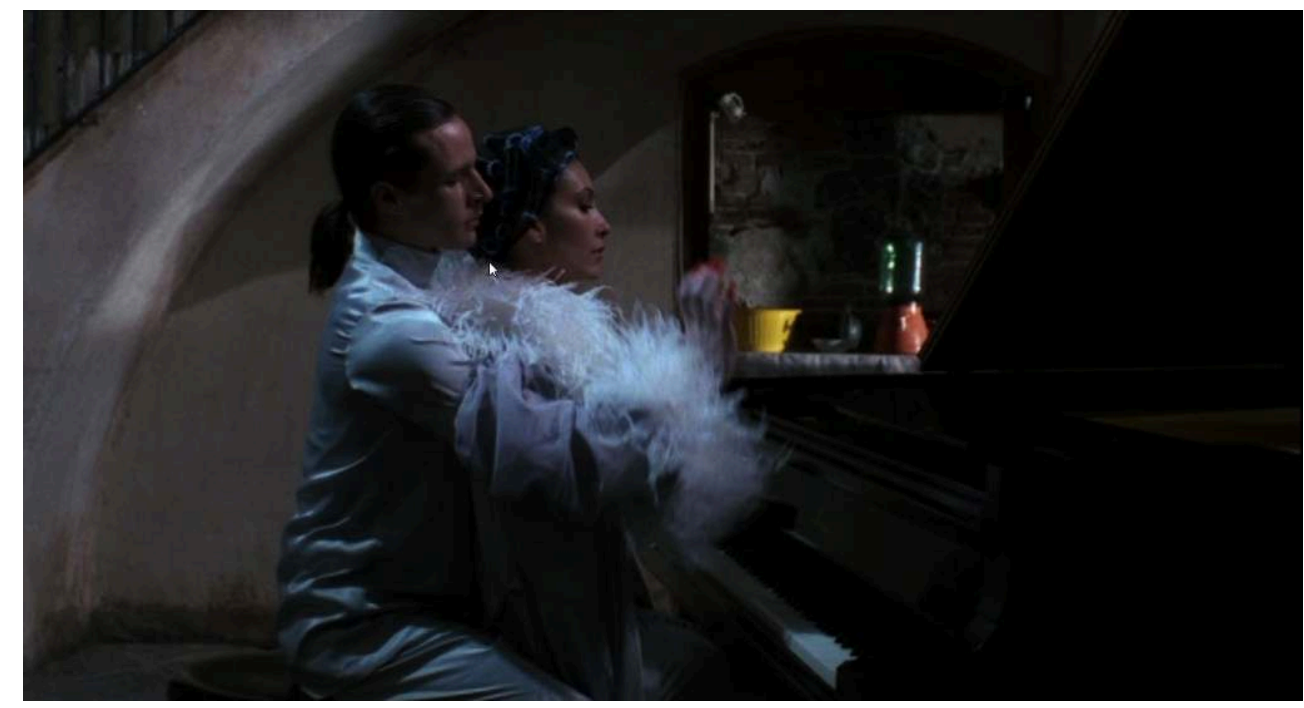

Concha et Fenix jouant au piano dans Santa Sangre, 1989

Doit-on rappeler la beauté de ces scènes de pantomime, en filiation directe avec le travail effectué en collaboration avec Marcel Marceau ? Au-delà de la prouesse visuelle qu'instaure à l'écran ce ballet entre ces deux personnages, cela a représenté un défi considérable en termes de mise en scène et de direction d'acteur. N'en témoigne cette citation de Jodorowsky quant aux méthodes employées pour rendre crédible cette double chorégraphie à l'écran :

On avait un problème de temps, parce qu'il [Axel Jodorowsky] ne connaissait pas l'actrice [Blanca Guerra], et il fallait que leur marche soit chronométrée, qu'ils soient comme un seul être. On a commencé à répéter, et j'ai bien vu que cela allait être long. Il était derrière elle et elle cachait ses bras dans son dos. Je lui ai dit de prendre le sexe et les testicules de Fenix dans ses mains et de le diriger comme ça, et c'est ce qu'on a fait : pendant tout le film, l'actrice a donc un pénis et des couilles dans sa main ! C'était ma méthode et elle a super bien marché ${ }^{20}$.

En plus de la domination de la marionnette sur son créateur à l'intérieur du scénario, l'actrice jouant Concha possédait un réel ascendant sur son partenaire en le guidant par la force, par le contrôle de sa virilité. Cette anecdote de tournage confirme l'idée de mainmise de la marionnette sur l'artiste, mais également de la mère sur son fils.

\section{Emprise maternelle et vulve procréatrice}

L'utilisation de l'animal comme signifiant symbolique de l'identité d'un personnage est un procédé régulièrement utilisé par Jodorowsky, lui qui avait affublé son héros d'un patronyme animalier dans $E l$ Topo $^{21}$. Ainsi dans l'œuvre jodorowskienne l'araignée peut être perçue comme une passerelle pour signifier la création artistique, mais elle est également un moyen d'amener des problématiques liées à l'acte de procréation et donc au lien de filiation. Contrairement à l'artiste Louise Bourgeois qui faisait de sa monumentale «Maman ${ }^{22}$ arachnéenne un abri rassurant et protecteur, Jodorowsky 
attribue lui aussi à l'araignée une dimension maternelle mais davantage tournée vers l'idée d'oppression d'un fils par rapport à l'omniscience de la mère. Cette association entre l'araignée et la figure maternelle se retrouve explicitement sous la plume de Jodorowsky qui écrit dans La Danse de la réalité : «Ma mère m'enlace d'abord avec deux, puis six et enfin huit bras : elle est à présent une tarentule ${ }^{23} »$. Une étreinte dont on comprend qu'il sera ardu de s'en extraire.

13 Il est possible d'effectuer ce même constat dans The Holy Mountain (La Montagne sacrée), sorti en 1973, où un vaste bestiaire est convoqué tout au long du film. Le récit porte sur une confrérie composée d'une somme de représentants de la société humaine et organisée autour d'un alchimiste, dont le but est de recueillir le secret de la vie éternelle. Ainsi, au moment de gravir la montagne comme ultime étape avant leur potentielle déification, les personnages se retrouvent associés à une pluralité d'animaux censés représenter leurs plus grandes peurs et frustrations. Affronter ces animaux sera l'ultime étape avant leur seconde naissance. L'araignée est partie constitutive de ce bestiaire et vient recouvrir le corps allongé et nu de Berg (Nicky Nichols), criant à pleins poumons face à cette multitude de tarentules. D'ailleurs, Jodorowsky lui-même évoque la portée symbolique d'une telle image : «Les araignées sont des symboles sexuels. Là, ce sont des tarentules. Les tarentules me terrifient ${ }^{24}$ ». Au-delà de la seule symbolique sexuelle, ces araignées prennent également une dimension maternelle lorsque l'on fait le lien avec la personnalité de Berg dépeinte un peu plus tôt dans le film.

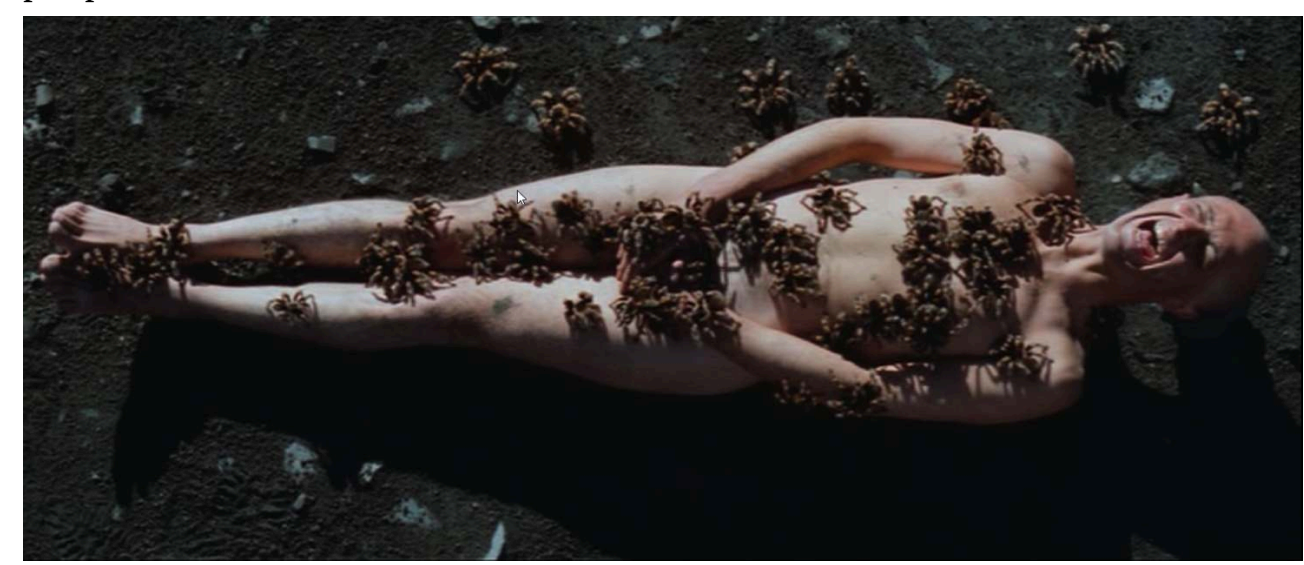

Berg recouvert de tarentules dans La Montagne sacrée, 1973.

14 Lors de ce portrait, on y voit le personnage s'adonnant à une relation incestueuse avec sa mère - ou la transposition de celle-ci. Elle est physiquement plus imposante et plus âgée que lui. Avec une fausse main, elle lui donne la fessée, gestuelle ultime de domination maternelle. Par la suite, on apprend que Berg se trouve être le conseiller financier d'un despote quelconque. Au moment de prodiguer ses conseils au politicien, il entre cérémonieusement dans une salle, relié à sa "mère " par un fil, que l'on peut assimiler soit au fil arachnéen soit au cordon ombilical. Telle une injonction maternelle, Berg annonce que pour sauver l'économie, il faut tuer quatre millions de citoyens en l'espace de six mois. Et tel un bon enfant obéissant, le chef d'État met immédiatement en œuvre les préconisations comme sous l'emprise d'un contrôle mental maternel translaté. 


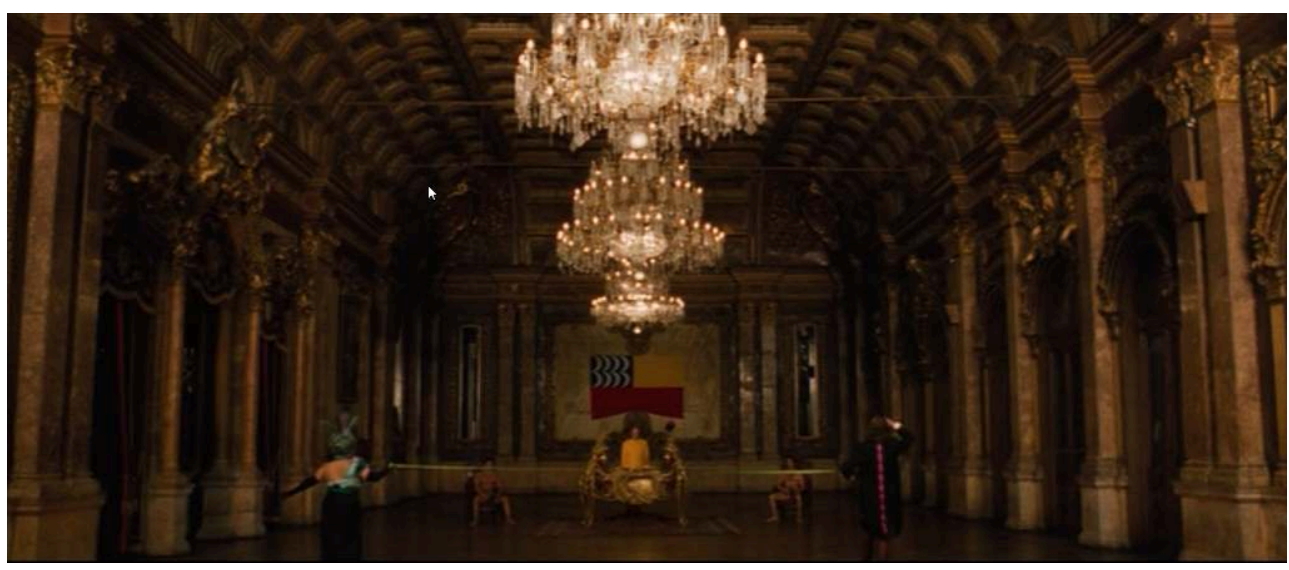

Berg et sa mère reliés par un fil dans La Montagne sacrée, 1973

Dans son premier long métrage Fando et Lis, Jodorowsky convoquait déjà cette image de l'araignée comme élément central de la psychologie du personnage principal et cela dès les premières séquences du film. Lorsque Lis (Diana Mariscal), allongée sur un sofa, se délecte d'une rose, en miroir c'est l'image de Fando (Sergio Kleiner) qui apparaît, jouant dans un premier temps aux petits soldats puis mettant le feu à une araignée empalée sur un pic. D'un point de vue symbolique, c'est une indication à propos de l'enfance avortée de Fando ainsi que de la relation néfaste qu'il entretient avec sa mère. En termes de montage cinématographique, cette image de l'araignée en feu ne souffre d'aucun manque de clarté quant à la signification à lui accorder. En effet, systématiquement ce plan ouvre et clôt toutes les résurgences mémorielles d'ordre familial que Fando convoquera lors de son voyage initiatique vers Tar.

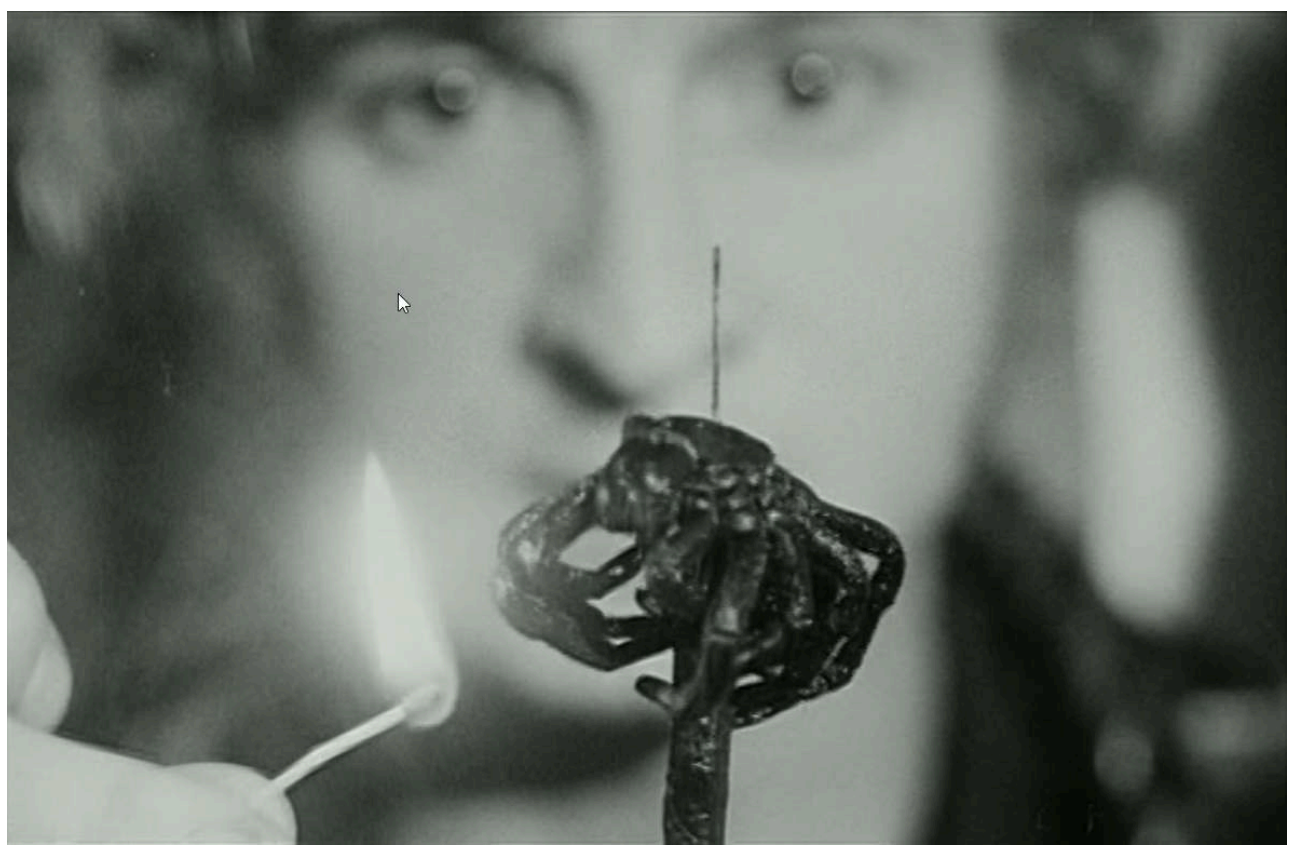

Fando brûlant une araignée dans Fando et Lis, 1968

La première occurrence de cette araignée en flamme précède un dialogue dans lequel le père de Fando envisage une multitude de futurs différents en réponse à son fils qui n'a de cesse de le soumettre verbalement à des amputations corporelles. Au pouvoir castrateur de la mère répond le pouvoir d'évasion poétique du père et de nouveau 
l'araignée en flamme fait son apparition comme personnification du problème existentiel qui animera Fando durant tout le film. Cette dualité parentale se retrouve lors de la séquence de la mort de la mère de Fando, une nouvelle fois introduite par le plan de l'araignée. Dans cette séquence onirique, la mère nourrit de force Fando avec des œufs qui, au-delà d'être attachés à l'image de l'embryon ou de l'organisme en gestation, apparaissaient au début du film, écrasés par des mains juste après la séquence de viol de Lis signifiant implicitement l'existence que l'on brise. Dans un second temps, on passe à la mort de la mère de Fando dont la dernière action sera de livrer en pâture son propre mari comme dissident révolutionnaire à des militaires s'empressant de l'exécuter. Ce passage précis est plutôt à mettre en lien avec l'histoire familiale de Fernando Arrabal, l'auteur de la pièce de théâtre, dont le père républicain a été dénoncé aux autorités franquistes au pouvoir. Enfin, dans un dernier élan, Fando en vient à tuer sa mère puis à l'enterrer. Comme une prédiction du meurtre de Lis à la fin du film, on a l'impression que seule la mort apporte de manière éphémère l'apaisement au personnage. Puis le plan de l'araignée fait alors une dernière apparition comme signe de l'accomplissement du désir de mort d'un fils envers sa propre mère. Un peu comme l'araignée-loup dévorée par sa propre progéniture, l'emprise maternelle infligée aux personnages de Jodorowsky se règle par la mise à mort symbolique de cet objet de frustration.

Plus de trente ans après Fando et Lis, Jodorowsky convoque de nouveau ces mêmes problématiques que sont l'emprise maternelle ainsi que le fils face à la mort de la mère. Santa Sangre est qualifié par son auteur de "tragédie psychanalytique ${ }^{25}$ ", dont le motif de l'aranéide semble absent. L'araignée apparaît dans une scène coupée du film, lorsque les deux parents, Orgo et Concha, se disputent la nature de l'enseignement à transmettre à Fenix. Le premier apprend le lancer de couteaux tandis que la seconde tente d'inculquer la foi religieuse envers la sainte manchote. C'est à ce moment qu'une tarentule apparaît dans le plan et est immédiatement tuée par le couteau d'Orgo, brisant par là-même l'effigie religieuse. Il est bien question ici de mise en scène symbolique d'une violence masculine exercée sur la mère, Jodorowsky allant même jusqu'à commenter cette scène comme la monstration d'un viol ${ }^{26}$. Malheureusement la seule réponse qu'apportera Concha à cette violence, c'est de la reproduire sur son fils, comme un signe d'impuissance teinté de vengeance.

18 À partir de ce moment-là, l'emprise de Concha sur Fenix ne fera que croître. En effet, c'est bien sous la pression des injonctions maternelles que Fenix est poussé au féminicide. Pour façonner son scénario, Jodorowsky s'est inspiré d'un véritable criminel mexicain dont il attribue la folie meurtrière à l'influence maternelle : « [...] je pense que ce sont les mères qui créent les tueurs en série ${ }^{27}$. " Une mère qui n'a pas besoin d'être vivante pour continuer à exercer son contrôle comme le figure la scène où Concha, depuis un sarcophage égyptien, incite son fils à l'assassinat lorsque celui-ci rétorque impuissant : "Vous l'avez entendue ? Elle veut que je vous tue de mes propres mains.» Telle une injonction venue d'outre-tombe, la domination maternelle n'a que faire de la mort et c'est bien cette idée que l'on retrouve dans une autre scène où Fenix et Concha interprètent une chanson au piano, reprenant ces paroles en chœur: «Maintenant que je t'ai perdu / Plus j'essaie de t'oublier / Et plus je pense à toi ». La séquence musicale se termine sur la mère et le fils qui s'embrassent de manière romantique, une relation incestueuse également éprouvée lors du tournage par l'acteur et l'actrice - Cristobal Jodorowsky et Blanca Guerra - afin d' ' approfondir leur rôle ${ }^{28}$. » 
19 L'élément salvateur pour Fénix est la réapparition du personnage d'Alma, perdue de vue au moment de la mort conjointe de ses parents au début du film. Il finit par renouer avec cette «âme » d'enfant qu'il avait oubliée. À la fin du film, Alma montre à Fénix la marionnette à l'effigie de sa mère. S'en suit une mise à mort symbolique de la mère dont la marionnette est jetée par une fenêtre puis, à l'instar de l'araignée de Fando, elle est finalement brûlée. C'est à ce moment précis que le spectateur assiste à la seconde naissance de Fénix à partir des cendres de la marionnette de sa mère. Cette mort symbolique est alors plus à même de résoudre les conflits intérieurs d'un individu que le décès véritable. Un tel procédé se retrouve dans Fando et Lis mais également au début d'El Topo, lorsque Alejandro Jodorowsky demande à son fils d'enterrer dans le sable du désert sa peluche ainsi que le portrait photographique de sa mère. Faire table rase de son lien filial pour instaurer la paix avec soi-même.

Que ce soit chez Karl Abraham dans son article "L'araignée, symbole onirique ${ }^{29}$ ou chez Sigmund Freud dans un passage de ses Nouvelles conférences d'introduction à la psychanalyse $e^{30}$ les deux hommes ayant en outre eu une importante correspondance ${ }^{31}$ l'image de l'araignée est analysée comme le symbole d'une mère redoutable et castratrice. Une telle signification fait écho au dimorphisme sexuel de la majorité des aranéides, la femelle étant généralement plus grande que le mâle, ainsi qu'à la pratique $\mathrm{du}$ cannibalisme après l'acte reproducteur par la femelle chez certaines espèces. Les deux psychanalystes interprètent également l'araignée comme une image du sexe féminin, faisant de l'arachnophobie une peur de la vulve. Jodorowsky formule une association similaire dans son roman de 1988, Enquête sur un chemin de terre, affirmant que les vagins de chair humaine renferment notamment « des tarentules au visage maternel ${ }^{32} »$. Par ailleurs, il utilise la figure d'Arachné pour l'affiche de Fando et Lis. En effet, celle-ci reprend une gravure de Gustave Doré illustrant La Divine Comédie et qui dépeint Arachné au Purgatoire, femme-araignée au corps convulsionné et aux pattes velues écartées. Cette fois, ce ne sont plus les poètes Dante et Virgile qui semblent fixer du regard l'organe génital de la tisseuse, mais Fando un tambour à la main, plus joyeux qu'apeuré.

21 Cette contemplation du corps arachnéen et du sexe féminin par une figure d'artiste suggère la métaphore sexuelle de la création ou, pour reprendre les mots de Jodorowsky : «la sexualité est la source de toute création ${ }^{33}$ ». La psychanalyste Hanna Segal établissait d'ailleurs un lien entre la créativité artistique et la dimension psychosexuelle génitale, écrivant que «créer une œuvre d'art est l'équivalent psychique d'une procréation ${ }^{34} »$. Cet écho entre création artistique et procréation est représenté dans un happening performé lors du festival de la libre expression de 1965, dont il existe des traces filmées. Comme il le relate dans le Théâtre de la guérison, Alejandro Jodorowsky met en scène sa naissance grâce à une femme couverte de satin noir, "une sorte de toile d'araignée ${ }^{35}$ " à laquelle est accroché un énorme vagin pneumatique. Il en déchire le fond et y prend refuge, le public assiste alors à l'accouchement symbolique de l'artiste. 


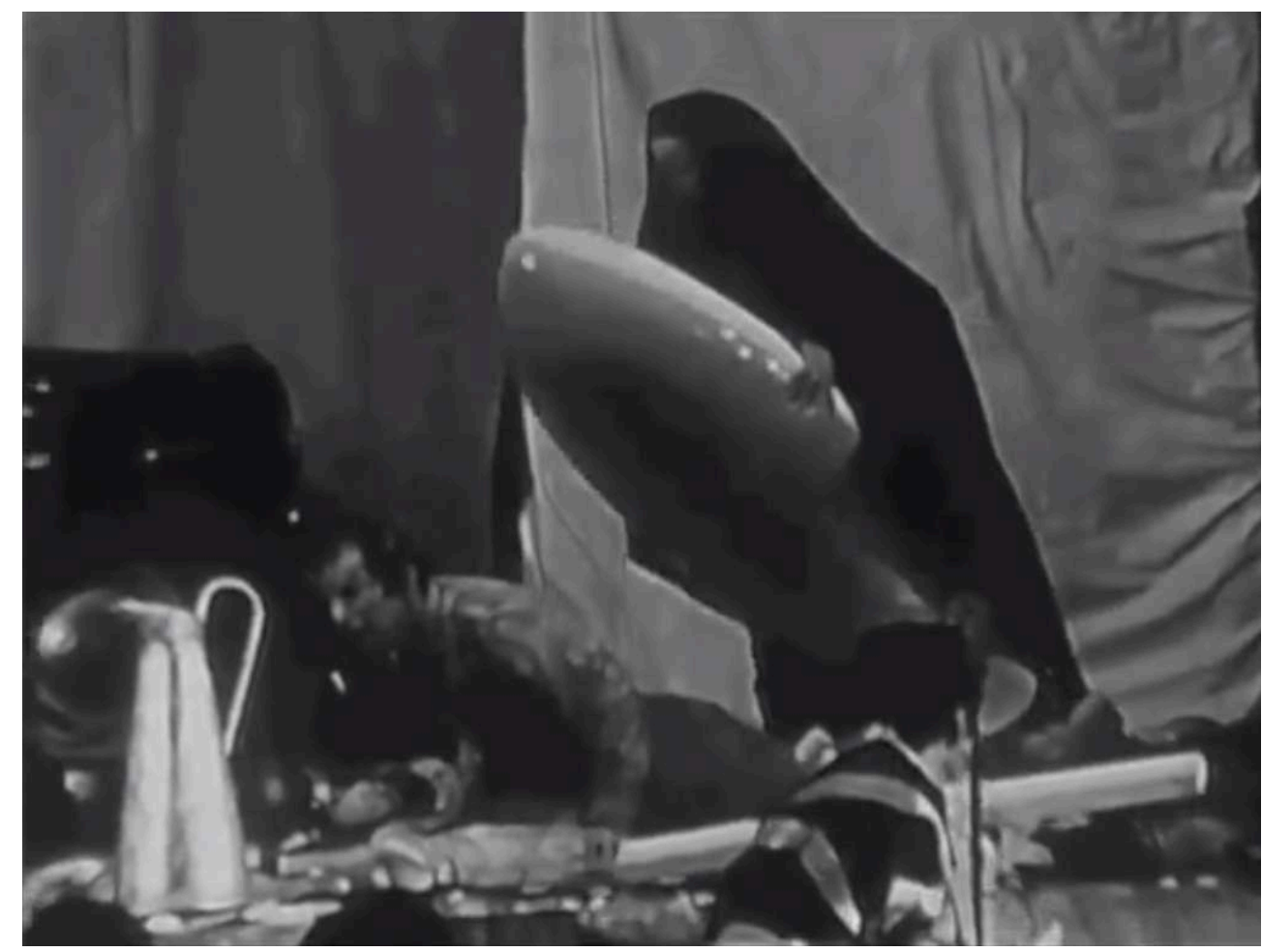

Jodorowsky dans un happening lors du festival de la libre expression de 1965

\section{Tisseuse de l'univers et créations oniriques}

Sans vouloir verser dans la psychanalyse appliquée, la convocation de telles sources trouve appui dans l'intérêt que Jodorowksy a eu pour cette discipline. La question de l'interprétation des rêves et de leur lien avec l'inconscient occupe une place notable dans sa pratique de la psychomagie. Jodorowsky donne d'ailleurs sa vision de l'art théâtral dans Le Théâtre de la guérison, un ouvrage exposant sa démarche thérapeutique et dans lequel il réfute l'approche psychologique réaliste. Cette fonction mimétique n'est pour lui qu'une réduction du monde à un aspect superficiel et fait «l'impasse sur la dimension inconsciente, onirique et magique de la réalité ${ }^{36} »$. De telles perspectives ne sont pas sans rappeler son lien avec les surréalistes, André Breton clamant dans le premier manifeste du groupe sa croyance dans « la résolution future de ces deux états, en apparence si contradictoires, que sont le rêve et la réalité, en une sorte de réalité absolue, de surréalitée ${ }^{37}$ ». Frédéric Aranzueque-Arriete fait de cet attrait pour l'inconscient une dimension importante de l'anti-mouvement Panique, hérité du surréalisme et du Postisme ${ }^{38}$. C'est par l'intermédiaire d'André Breton que Jodorowsky découvre un ouvrage qui devait le marquer, Le Rêve et les moyens de les diriger d'Hervey de Saint Denis ${ }^{39}$. Il expérimente alors cette possibilité de devenir le maître de ses rêves et d'en contrôler les images. Cela renvoie au pouvoir démiurgique évoqué précédemment, convoquant de nouveau un parallèle avec la figure divine :

Dans le rêve lucide, on se veut démiurge, on usurpe le rôle de Dieu. Dans le rêve humble, par contre, on renonce à tout contrôle, on ne court plus après le pouvoir. On n'éprouve plus le désir d'être un guerrier, de façonner son destin. On comprend que l'inconscient est un univers au sein duquel on a à jouer parfaitement un petit rôle ${ }^{40}$. 
Psychomagie, autre ouvrage traitant de son approche thérapeutique, cette relation entre puissance, création et divinité est explicitée à travers l'exemple du totem animal. Faisant référence à des pratiques ancestrales qui, selon lui, trouvent encore un écho à l'heure actuelle, Jodorowsky voit dans cette association entre corps humain et animal une façon de se rendre maître de ce dernier tout en lui accordant un statut divin. Il s'agit là d'une démarche créatrice qui a d'ailleurs nourri diverses mythologies ainsi que les productions artistiques correspondantes.

C'est ainsi, au début, que l'être humain a produit sa créativité. De chaque chose qu'il incorpore il fait un dieu. Notre être croît avec chaque dimension incorporée. Après avoir incorporé l'animal, l'homme devient chasseur; il peut élever des vaches, des moutons... S'il incorpore un tigre, il peut chasser un tigre ; s'il introduit un éléphant, il peut dompter un éléphant. De là vient le dieu Ganesh en Inde, avec sa tête d'éléphant ${ }^{41}$.

Mais surtout, la suite de cet extrait renvoie au thème de la création du monde et l'animal associé à un tel concept se trouve être l'araignée :

Pour la culture indienne, l'araignée est Maya, celle qui tisse l'univers ; et cet univers est un rêve, un rêve tissé en forme de toile d'araignée. Dans le Tarot nous voyons que l'arcane 8 est la Justice, celle-ci étant une descendante de l'araignée. Tout 8 descend de l'araignée : les huit pattes, le symbole de l'infini et autres références ${ }^{42}$.

L'analogie entre création cosmique et figure arachnéenne est certes présente dans les Upanishad, textes sacrés de l'hindouisme, mais se retrouve également dans plusieurs mythes. Pour les Kayah de Bornéo, la divinité primordiale est une araignée tissant une toile dans laquelle tombe une pierre qui devient la terre ${ }^{43}$. Chez les Amérindiens Hopis, la créatrice de l'humanité est Koyangwuti, la grand-mère araignée qui façonne les humains à partir d'argile ${ }^{44}$. Il y a l'araignée Sussistinnako des Sia qui trace deux lignes, l'une reliant le nord au sud, l'autre l'est à l'ouest et, se mettant à chanter, elle fait émerger les deux premières femmes ${ }^{45}$. Dans un mythe d'Asie septentrionale, Kúrguñéut, une petite femme-araignée, descend sur terre à l'aide de son fil, faisant éclater son ventre pour donner naissance aux quatre premières femmes. Sans parcourir davantage les mythologies du monde qui donnent à l'araignée un rôle anthropogonique majeur, l'autre élément qui ressort de la citation de Jodorowsky est la dimension onirique de l'univers. Il renvoie à la déité hindou-bouddhiste Mâyâ qui symbolise l'illusion, l'homme réduisant le cosmos à ce qu'il peut percevoir, reprenant cette idée évoquée précédemment qu'on ne peut pas se satisfaire seulement du visible. Ce qu'il y a d'intéressant dans la doctrine de Mâyâ, notamment développée par Gaudapâda, c'est qu'elle rejette les objets extérieurs comme des entités existantes pour affirmer que tout n'est que création de l'esprit ${ }^{46}$. Derrière le thème du rêve, c'est la question de l'imagination créatrice qui est en jeu.

De telles conceptions sont présentes dans l'œuvre de Jodorowsky, et plus particulièrement dans une bande dessinée éditée en trois tomes en 2010 et 2012 et dont il est le scénariste. Mise en image par Nicolas Fructus, Showman Killer ${ }^{47}$ suit l'aventure d'un assassin créé par un généticien, qui finit par protéger un jeune garçon. Dans leur quête, ils font la rencontre des trois onironautes, qui se situent entre rêve et réalité, contrôlant les mondes créés par l'imagination humaine afin de protéger l'univers du chaos. Ainsi, cette dualité entre le domaine de l'inconscient et la matière perceptible qui n'en est pas une, puisque ces deux plans sont connectés, est au cœur de l'histoire de Showman Killer. Comme le disent les onironautes, « le monde onirique est aussi réel que le monde de l'éveil et nous sommes le pont qui unit ces deux mondes ${ }^{48} . .$. » Or, le danger 
vient d'un rêveur, le bouffon qui se projette en personnage puissant, ouvrant alors une faille vers une autre dimension. Jodorowsky représente véritablement le pouvoir de l'imagination. Le traitement visuel de ce passage est également intéressant: non seulement leur rencontre n'est possible qu'après la traversée d'un labyrinthe, image proche de la toile d'araignée, mais les protagonistes se trouvent sur la bonne voie après s'être engouffrés dans une vulve tracée par Ibis. Celle-ci n'est que la matérialisation de ses trois mamamans qui, pareilles aux Parques, guident la destinée des personnages. En outre, les onironautes sont reliées entre elles par des câbles au niveau de leur nuque, de leur nombril et de leur vagin.

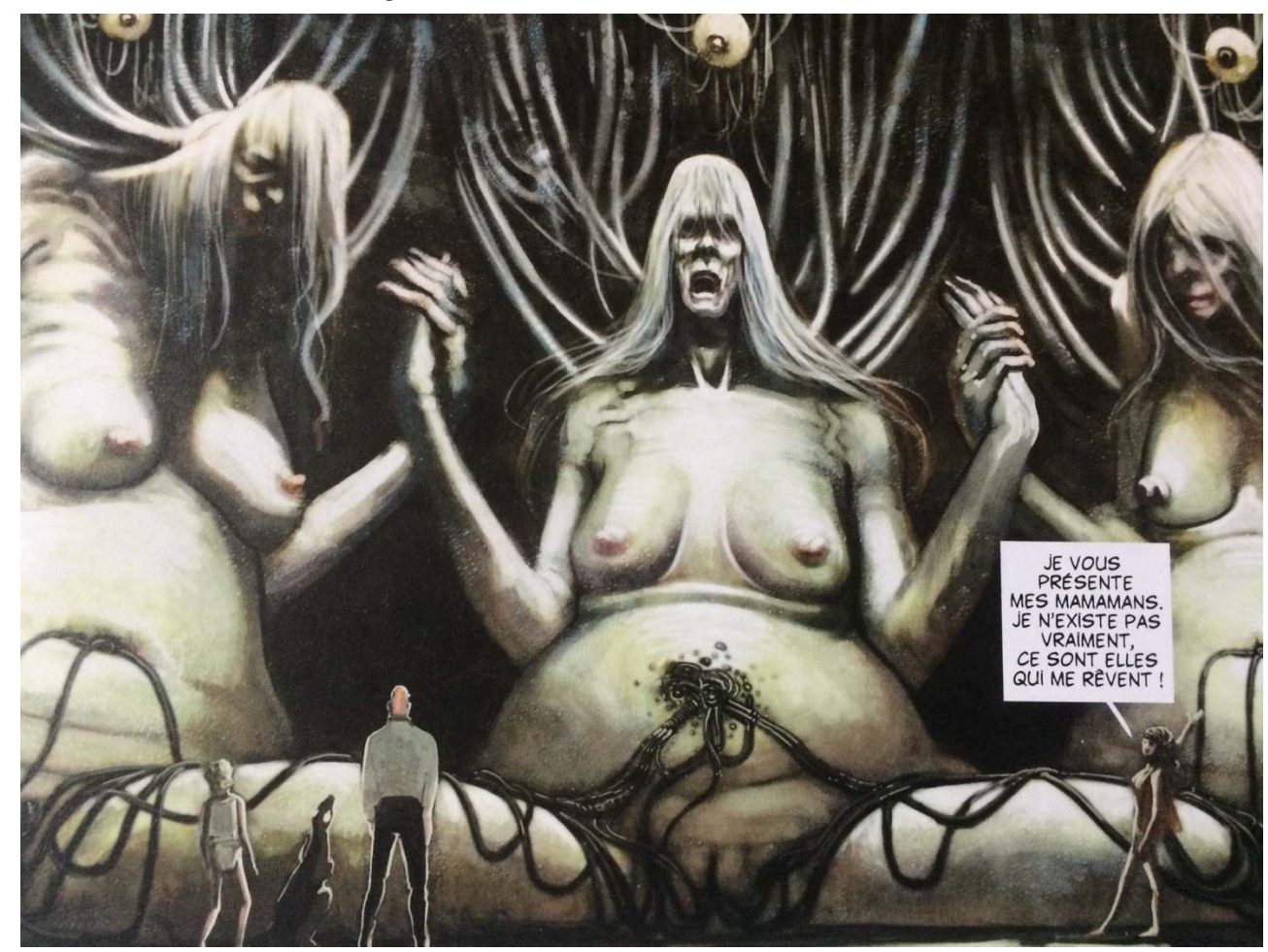

Les onironautes par Nicolas Fructus dans Showman Killer, tome 2, 2012

Cette esthétique du réseau, lien entre différents mondes et qui prend forme par l'entremêlement de fils de connexion entre en résonance avec la toile d'araignée. Il n'est pas étonnant de trouver dans Les Technopères ${ }^{49}$, autre bande dessinée dont le premier tome est publié en 1998, l'image d'une cyberentule, araignée technologique qui vit dans la jungle des câbles du système informatique. Là encore, Jodorowsky met en place différents niveaux de réalité, avec un monde virtuel qui fascine le jeune héros Albino, aspirant à devenir un créateur de jeux. Alors qu'il propulse son esprit dans l'ordinateur, il doit échapper aux attaques de monstres liées à l'anti-virus, dont fait partie la cyberentule. Après avoir vaincu ces obstacles, il arrive devant un œuf blanc qui lui ouvre la porte d'un endroit secret. Les Technopères est une série dérivée de l'univers de sa première saga intitulée $L^{\prime} I n c a l^{50}$ réalisée avec Mœbius, dont la collaboration sur Dune ${ }^{51} n^{\prime}$ avait pas pu se concrétiser. C'est dans le deuxième tome de cette série, paru en 1982, que les deux artistes nous offrent une planche en pleine-page, figurant une construction technologique sous la forme d'une araignée. Celle-ci est le fruit des Techno-technos, secte scientifico-religieuse dirigée par le Techno-pape et qui voue un culte aux ténèbres. Alors que l'anti-héros de la série, John Difool, est prisonnier de cette structure arachnéenne, l'abdomen de cette construction se détache en un œuf 
d'ombre dont la finalité est d'aspirer tous les photons de la galaxie. Son fidèle compagnon ailé Deepo veut le libérer et fonce alors sur la forme ovoïde noire qui surmonte la tête du Techno-pape, décrite comme son "psycho-abdomen » dont il tire ses pouvoirs psychiques. Jodorowsky donne encore à voir ici l'action des forces mentales sur la réalité matérielle. Le symbolisme de l'araignée et de l'œuf est ici renversé, puisqu'il ne s'agit plus de création mais de destruction de l'univers.

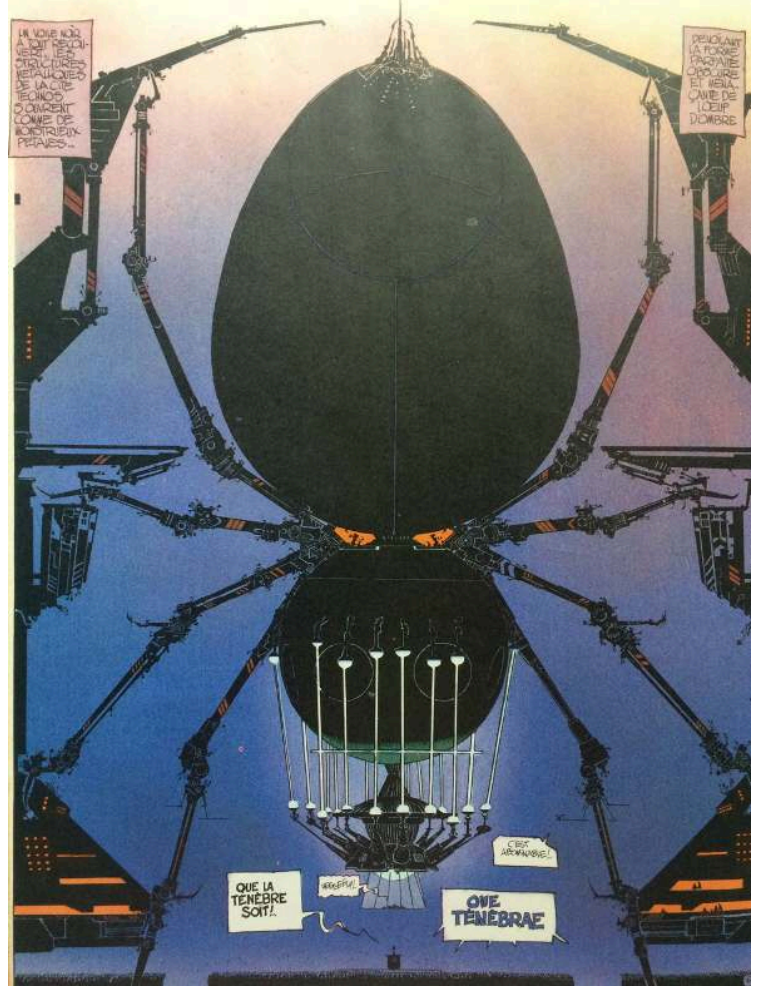

L'œuf d'ombre par Mœbius dans L'Incal, t. II, 1982

\section{Conclusion}

Du théâtre à la psychomagie, du cinéma à la littérature et à la bande dessinée, Jodorowsky construit une même approche, utilisant la puissance expressive des symboles pour toucher l'individu au plus profond de son être, comme il pouvait l'écrire : « l'art doit libérer les forces de l'inconscient et doit travailler comme une clé dans votre inconscient ${ }^{52} »$. Le symbolisme de l'araignée met en jeu l'image du créateur dans ses différentes acceptions : artiste orgueilleux, figure divine, marionnettiste dont la créature peut lui échapper, mère procréatrice, rêveur plus ou moins lucide ou encore inventeur de mondes virtuels. Ces multiples représentations arachnéennes puisent tout autant dans les récits mythologiques ou philosophiques sans restriction spatiale, que dans la psychanalyse ou bien la propre imagination de l'auteur. Leur analyse permet d'appréhender la question de la création dans toute sa richesse, qu'elle soit artistique, génésique, anthropogonique ou onirique. 


\section{NOTES}

1. Alejandro Jodorowsky, Cabaret Mystique, Paris, Albin Michel, 2008, p. 9-10.

2. Ferdinand de Saussure, Cours de linguistique générale, Wiesbaden, édition critique par Rudolf Engler, 1967, cité dans Tzvetan Todorov, Théories du symbole, Paris, Éditions du Seuil, 1977, p. 355.

3. Émilie Granjon, «Le Symbole : une notion complexe», Protée, vol. 36, n¹, printemps 2008, p. 17-28.

4. Alejandro Jodorowsky, «Les araignées sans mémoire », dans Les Araignées sans mémoire et autres fables paniques, Paris, Les Humanoïdes Associés, 1980, p. 17-19.

5. Sylvie Ballestra-Puech, Métamorphoses d'Arachné: l'artiste en araignée dans la littérature occidentale, Genève, Droz, 2006, p. 339.

6. Alejandro Jodorowsky, «L'araignée ambitieuse ", dans Les Araignées sans mémoire et autres fables paniques, op. cit., p. 135.

7. Alejandro Jodorowsky, Fables paniques, Arles, Actes Sud, 2017, p. 88.

8. Alejandro Jodorowsky, «L'araignée ambitieuse », op. cit., p. 135.

9. Sylvie Ballestra-Puech, Métamorphoses d'Arachné: l'artiste en araignée dans la littérature occidentale, op. cit., p. 340.

10. Caroline Combronde, «Les platoniciens de l'art à la Renaissance », Revue Philosophique de Louvain, tome 97, n 2, 1999, p. 273-276.

11. Gilles Farcet, Alejandro Jodorowsky, La Tricherie sacrée, Paris, Dervy, 1989, p. 65.

12. Ovide, «Pallas et Arachné », Métamorphoses, Livre VI, 1-145.

13. Alejandro Jodorowsky, Fables paniques, op. cit., p. 166.

14. Ibid.

15. Jean-Paul Coillard, Alejandro Jodorowsky, De la cage au grand écran: entretiens avec Alejandro Jodorowsky et analyse de son univers cinématographique, Paris, K-ïnite éd., 2009, p. 77.

16. Commentaires audio d'Alejandro Jodorowsky sur Fando et Lis, dans L'Univers de Alejandro Jodorowsky [DVD], Wild Side Video, 2008.

17. Elisabeth Pouilly, Le Monstrueux et le sacré dans les œuvres théâtrales et cinématographiques d'Alexandro Jodorowsky, mémoire de master, université de Toulon, 2010, p. 18, en ligne sur HAL.

18. Alejandro Jodorowsky, Le Théâtre de la guérison, Paris, Albin Michel, 2001, p. 49-50.

19. L'Homme invisible (The Invisible Man), James Whale, États-Unis, 1933.

20. Jean-Paul Coillard, Alejandro Jodorowsky, De la cage au grand écran : entretiens avec Alejandro Jodorowsky et analyse de son univers cinématographique, op. cit., p. 256.

21. Le mot espagnol « topo » désigne en français une taupe.

22. Louise Bourgeois, Maman, 1999, sculpture en bronze, 10 × 9 m, Musée des beaux-arts du Canada, Ottawa.

23. Alejandro Jodorowsky, La Danse de la réalité, Paris, Albin Michel, 2004, p. 79.

24. Commentaires audio d'Alejandro Jodorowsky du film La Montagne sacrée, dans L'Univers de Alejandro Jodorowsky [DVD], op. cit.

25. Jean-Paul Coillard, Alejandro Jodorowsky, De la cage au grand écran : entretiens avec Alejandro Jodorowsky et analyse de son univers cinématographique, op. cit., p. 262.

26. Commentaires audio d'Alejandro Jodorowsky sur deux scènes coupées de Santa Sangre, dans Santa Sangre [DVD], Wild Side Video, collection « Les Introuvables », 2013.

27. Jean-Paul Coillard, Alejandro Jodorowsky, De la cage au grand écran: entretiens avec Alejandro Jodorowsky et analyse de son univers cinématographique, op. cit., p. 254.

28. Ibid., p. 256.

29. Karl Abraham, «L'Araignée comme symbole onirique », Cuvres complètes, t. II, Paris, Payot, 1965, p. 146-150. 
30. Sigmund Freud, Nouvelles conférences d'introduction à la psychanalyse, [1933], Paris, Gallimard, 1984 , p. 36.

31. Karl Abraham, Sigmund Freud, Correspondance : 1907-1926, Paris, Gallimard, 1969.

32. Alejandro Jodorowsky, Enquête sur un chemin de terre, Paris, Albin Michel, 2018, p. 28.

33. Gilles Farcet, Alejandro Jodorowsky, La Tricherie sacrée, op. cit., p. 80.

34. Jean-Michel Quinodoz, A l'écoute d'Hanna Segal. Sa contribution à la psychanalyse, Paris, Presses Universitaires de France, 2008, p. 34.

35. Alejandro Jodorowsky, Le Théâtre de la guérison, op. cit., p. 76-77.

36. Ibid.p. 50.

37. André Breton, Manifestes surréalistes, Paris, Gallimard, coll. « Folio Essai », 1962, p. 60.

38. Frédéric Aranzueque-Arriete, Panique: Arrabal, Jodorowsky, Topor, Paris, L'Harmattan, 2008, p. 28-29.

39. Alejandro Jodorowsky, Le Théâtre de la guérison, op. cit., p. 90.

40. Gilles Farcet, Alejandro Jodorowsky, La Tricherie sacrée, op. cit., p. 97-98.

41. Alejandro Jodorowsky, Psychomagie, Paris, Albin Michel, 2019, p. 196-197.

42. Ibid.

43. Jean-Loïc Le Quellec et Bernard Sergent, Dictionnaire critique de mythologie, Paris, CNRS éditions, 2017, p. 78-81.

44. David Adams Leeming, Creation myths of the world an encyclopedia, vol. I, Santa Barbara, ABCCLIO, 2010, p. 131.

45. Alexander Hartley Burr, The Mythology of all races. North American, Volume X, Boston, Marshall Jones Company, 1916, p. 202-203.

46. Daryush Shayegan, Hindouisme et soufisme, Paris, Albin Michel, 1996, p. 79.

47. Nicolas Fructus, Alejandro Jodorowsky, Showman Killer, tome 2: L'enfant d'or, Paris, Éditions Delcourt, coll. « Neopolis », 2011.

48. Ibid., p. 42.

49. Fred Beltran, Zoran Janjetov, Alejandro Jodorowsky, Les Technopères, tome 1: Planeta Games, Genève, Les Humanoïdes associés, 2003, p. 7.

50. Alejandro Jodorowsky, Moebius, L'Incal : une aventure de John Difool, tome 2 : L'Incal Lumière, Les Humanoïdes Associés, coll. «Eldorado », 1986.

51. Alejandro Jodorowsky a commencé à travailler sur la première adaptation cinématographique du roman de Frank Herbert, Dune. Mœbius avait participé à ce projet inachevé en réalisant plusieurs planches de storyboard. Ces informations sont visibles dans le documentaire américain Jodorowsky's Dune de Frank Pavich, sorti en 2013.

52. André Leroux, "Alexandro Jodorowsky: "Mon cinéma est métaphysique " ", Le Devoir, 2 février 1974, p. 18.

\section{RÉSUMÉS}

L'étude du motif arachnéen permet de tisser des liens entre les différentes formes artistiques de l'œuvre de Jodorowsky. En dépit de la diversité de médiums, des thématiques communes émergent et permettent d'articuler fables, théâtre, cinéma, happening et bandes dessinées au sein d'un même discours. En effet, la récurrence de l'image d'arachnides ouvre sur la question de la création, renvoyant à des figures symboliques conceptrices. 
The study of spider motif forges links between the different artistic forms of Jodorowsky's work. In spite of the diversity of mediums, common themes emerge and fables, theater, cinema, happening and comics can be articulated in the same discourse. Indeed, the recurrence of the image of arachnids opens on the question of creation, referring to symbolic designer figures.

\section{AUTEURS}

\section{JULES FONTAINE}

Ancien président de la cinémathèque de Bourgogne-Jean Douchet, Jules Fontaine est titulaire d'un master en histoire du cinéma à Panthéon-Sorbonne. Il a travaillé sur le film Haut les mains ! de Jerzy Skolimowski, puis sur l'érotisme dans l'œuvre de Walerian Borowczyk, sujet sur lequel il a pu proposer plusieurs conférences. Ses activités se tournent désormais vers la création audiovisuelle, avec plusieurs projets en préparation, notamment le podcast Loulou et la brigade cinéphile dont la première saison est disponible à l'écoute.

\section{MARION SERGENT}

Doctorante en histoire de l'art contemporain à Sorbonne-Université, Marion Sergent termine actuellement sa thèse intitulée Les Artistes musicalistes : théories et pratiques d'une union des arts, groupe sur lequel elle a écrit un article visible sur fabula.org. Lauréate 2018 de la bourse de recherche de l'école des modernités de la fondation Giacometti, ses prochaines publications s'intéressent pour l'une à la peinture musicale de Gustave Bourgogne, actes du colloque à paraître aux Éditions L'Harmattan, pour une autre à la question de l'ornement chez Jean d'Udine au sein du numéro 23 de la Nouvelle Revue d'Esthétique. 